\title{
STUDY ON THE TRAJECTORY OF FREE-END FIBER IN JET VORTEX SPINNING BASED ON THE ELASTIC THIN-ROD FINITE ELEMENT MODEL OF FLEXIBLE FIBER
}

\author{
Chenchen Han, Weidong Gao'* ${ }^{1 *}$ Lifen Chen $^{2}$ \\ ${ }^{1}$ School of Textile and Clothing, Jiangnan University, Wuxi, Jiangsu 214122, China; \\ ${ }^{2}$ Jiangsu Sunshine Group Co., Ltd., Jiangyin, Jiangsu 214426, People's Republic of China \\ Corresponding author, Weidong Gao, gaowd3@163.com
}

\begin{abstract}
:
During the air flow twisting process of jet vortex spinning, the moving characteristics of flexible free-end fiber are complex. In this paper, the finite element model of the fiber is established based on elastic thin rod element. According to the air pressure and velocity distribution in the airflow twisting chamber of jet vortex spinning, this paper analyzes the undetermined coefficients of the finite element kinetic differential equation of the free-end fiber following the principle of mechanical equilibrium, energy conservation, mass conservation and momentum conservation. Based on numerical simulation, this paper gets the trajectory of the free-end fiber. Finally, the theoretical result of the freeend fiber trajectory by finite element simulating is tested by an experimental method. This paper has proposed a new method to study the movement of the fiber and learn about the process and principle of jet vortex spinning.
\end{abstract}

\section{Keywords:}

Jet vortex spinning, flow field characteristics, finite element model; movement of fiber, trajectory of fiber

\section{Introduction}

Jet vortex spinning twists the free-end fiber at the hollow spindle inlet by high-speed rotating air. Its high spinning speed, high degree of automation, short process, low energy consumption and excellent yarn performance have got the industry's attention, which make the jet vortex spinning have a wide range of market prospects. ${ }^{[1]}$ Basal and Oxenham ${ }^{[2]}$ analyzed the correlation of the jet vortex spinning process parameters and the fiber transfer in the pure cotton jet vortex spun yarn. Tyagi et al. ${ }^{[3]}$ analyzed the influence of the nozzle pressure, the distance between former roller jaw and hollow spindle inlet and the spinning speed on the yarn structure classification and the fiber composition ratio of the polyestercotton jet vortex spun yarn by an experimental method. $\mathrm{Li}^{[4]}$ designed the parameters of the Murata Vortex Spinning (MVS) nozzle, and the number of nozzle holes was increased to six. The optimum nozzle parameters of the pure cotton jet vortex spun yarn were determined, and the influence of the nozzle structure parameters on the yarn forming and yarn performance was discussed by the experimental method. Yuan et al. ${ }^{[5]}$ qualitatively analyzed the correlation of the hollow spindle cone angle, the free-end fiber lodging state and the jet vortex spun yarn structure. Ortlek et al. ${ }^{[6]}$ discussed the effect of the hollow spindle inner diameter and the hollow spindle usage on the $100 \%$ viscose MVS yarn performance. Liu and $\mathrm{Xu}^{[7]}$ analyzed the correlation of the jet vortex spinning airflow intensity and the jet vortex spun yarn twist strength. Guo et al. ${ }^{[8]}$ analyzed the correlation of the hollow spindle cone angle and the jet vortex spinning flow field distribution by the twodimensional standard turbulent flow field model. Zou et al. ${ }^{\left[{ }^{[9}\right.}$ analyzed the correlation of the hollow spindle inner diameter, the jet vortex spinning airflow velocity and the jet vortex spun yarn structure by the three-dimensional standard turbulent flow field model.

The twisting airflow chamber of jet vortex spinning is irregular, non-visible and small size, and so it is difficult to catch the free-end fiber moving images in the flow field. At present, the domestic and foreign scholars are mainly based on the flow field simulation and experimental methods to study the yarn mechanism of jet vortex spinning and the relationship between yarn forming process and yarn structure, indirectly and qualitatively. In this paper, we established the threedimensional finite element model of fiber, based on the elastic thin rod element and the dynamic characteristics of the free-end fiber flexible body during jet vortex spinning process. Combined with the distribution characteristics of flow field air pressure and velocity in the twisting airflow chamber of jet vortex spinning, we established the finite element kinetic differential equation of the free-end fiber flexible body and analyzed the undetermined coefficients of the finite element kinetic differential equation, following the principles of mechanics balance, energy conservation, mass conservation and momentum conservation. Then, we could get the trajectory of the free-end fiber flexible body by numerically simulating the movement law of the free-end fiber under the high-speed rotating airflow in the twisting airflow chamber of jet vortex spinning. Finally, we carried out experiments to test the theoretical results of the finite element simulated fiber trajectory. In this paper, the elastic thin rod element model is used to simulate the trajectory of free-end fiber under the high-speed rotating airflow in the twisting airflow chamber of jet vortex spinning, which is a new research method to study the process and principle of jet vortex spinning. 


\section{Establishment and dynamics analysis of the single fiber finite element model}

Until now, in the study about the yarn mechanism of various spinning technologies, the performance and the movement of the fiber under complex forces need to be understood urgently. The fiber is a kind of elongated body with high aspect ratio, elasticity and flexibility. The actual movement law of the free-end fiber in the high-speed twisting airflow field is very complicated, involving translation, rotation, bending, stretching and so on. In the previous study, in order to facilitate the calculation, the fiber is often simplified into a relatively simple mechanical model. For example, the non-spherical rigid particle fiber model, multirigid body chain fiber model and so on ${ }^{[10]}$ generally take the fiber as a space curve, regardless of the torsional deformation on the fiber section, which cannot directly and quantitatively characterize the real movement law of the fiber in the airflow field. In this paper, according to the characteristics of the freeend fiber flexible body in the twisting airflow chamber of jet vortex spinning, we take the fiber as a continuous elastic thin rod. The nonlinear mechanics methods generally use simultaneous partial differential equations with multi-parameters to express the small linear behavior of elastic thin rods, which are difficult to practically calculating three-dimensional space problems. ${ }^{[11]}$ In this paper, we used the method of stepwise loading and successive approximation to calculate the space mechanics problem of the elastic thin rod, based on the classical rigid body dynamics study. Then, we numerically simulated the nonlinear large deformation problem of the free-end fiber, combined with its apace movement characteristics in the twisting airflow chamber of jet vortex spinning.

\subsection{Establishment of the single fiber finite element model}

In this study, the elastic thin rod is used as the basis of a fiber model. The whole single fiber is divided into $n+1$ independent microsegments, and a node is arranged at the center of the cross-section on each microsegment midpoint. Each node on the cross-section is consolidated into a motion coordinate system, which is the node coordinate system. The inertial reference system, which is fixed on the ground, is used as the total coordinate system for the finite element analysis. The elastic thin rod finite element model in the overall space is shown in Figure 1. The coordinate system of each node has six degrees of freedom relative to the overall coordinate system. By setting six coordinate parameters, the relative position of the node can be determined to describe the moving line displacement. Considering the quality of the elastic thin rod, we took the quality of each microsegment as a rigid body, which generally moves with the node coordinate system. Considering the elasticity of the elastic thin rod, each of the two adjacent nodes is considered to be connected by a space elastic thin rod unit, which considers the flexibility only and does not consider the quality. All the external forces acting on the elastic thin rods are simplified to their nodes. The infinitesimal rigid segment of the elastic thin rod finite element model is shown in Figure 2 , which generally moves with the node coordinate system. In Figure 2, $O-x y z$ is the fixed total coordinate system; $o^{\prime}-u v w$ is the motion coordinate system fixed on the infinitesimal rigid segment $i$, which is the node coordinate system. The origin of the node coordinate system is the infinitesimal rigid segment centroid; $u$ axis is the axis direction of the infinitesimal rigid segment; $v$ and $w$ axes are located on the cross-section, which has the centroid of the infinitesimal rigid segment.

\subsection{Dynamics analysis of the single fiber finite element model}

In order to achieve the feasibility and rationality of the calculation about the nonlinear large deformation problem of the free-end fiber, we have made some simplifications and assumptions in this study as follows: (1) do not consider the reaction of the fiber to the airflow; (2) ignore the interaction between the fiber, the central strip and the inner wall of the cavity; (3) do not consider the tensile elasticity and the torsional elasticity of the fiber; (4) the free end of the fiber already exists when it is under the airflow twisting; and (5) theoretically, the force of the airflow field is continuously distributed on the fiber surface.

According to the centroid motion theorem, ${ }^{[12]}$ the kinetic motion differential equation of the infinitesimal rigid segment is as follows:

$$
[\boldsymbol{m}]\left\{\begin{array}{l}
a_{x} \\
a_{y} \\
a_{z}
\end{array}\right\}=\left\{\begin{array}{l}
F_{x} \\
F_{y} \\
F_{z}
\end{array}\right\}
$$

Where, $m$ is the mass of the infinitesimal rigid segment; $\left(a_{x}, a_{y}, a_{z}\right)$ is the absolute acceleration of the centroid relative to the fixed total coordinate system; and $\left(F_{x}, F_{y}, F_{z}\right)$ is the main of the external force vector on the infinitesimal rigid segment.

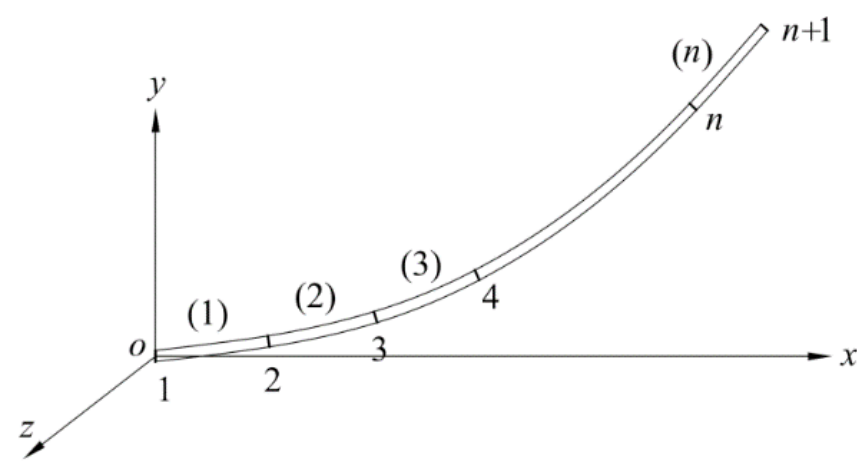

Figure 1. The elastic thin rod finite element model in the overall space.

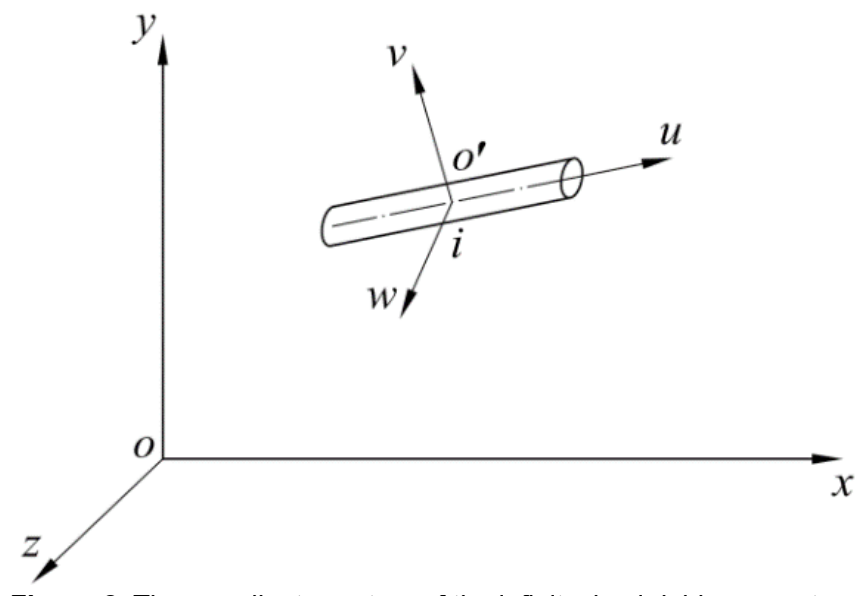

Figure 2. The coordinate system of the infinitesimal rigid segment. 
Converting the freedom degree of the infinitesimal rigid segment fixed on each node into the freedom degree of the fixed total coordinate system and then expressing the kinetic motion differential equation as follows:

$$
\left[\boldsymbol{m}^{b}\right]_{6(n+1) \times 6(n+1)}^{i}\{\boldsymbol{a}\}_{6(n+1) \times 1}+\left\{\boldsymbol{R}^{b}\right\}_{6(n+1) \times 1}^{i}=\left\{\boldsymbol{F}^{b}\right\}_{6(n+1) \times 1}^{i}
$$

Where, the vacancy element is zero; $\{\boldsymbol{a}\}_{6(n+1) \times 1}$ is the acceleration of all the nodes.

Converting the freedom degree of the external force on the elastic thin rod into the freedom degree of the fixed total coordinate system and then expressing the external force equation as follows:

$$
\{\boldsymbol{F}\}_{6(n+1) \times 1}^{e}=[\boldsymbol{k}]_{6(n+1) \times 6(n+1)}^{e}\{\boldsymbol{\delta}\}_{6(n+1) \times 1}^{e}
$$

Where, $\{\boldsymbol{F}\}^{e}$ is the external force on the elastic thin rod relative to the fixed total coordinate system $O-x y z ;[\boldsymbol{k}]^{e}$ is the element stiffness matrix of the elastic thin rod relative to the fixed total coordinate system $O-x y z$.

$\left\{F^{b}\right\}_{6(n+1) \times 1}^{i}$ in equation (2) is considered as the external force required for each infinitesimal rigid segment and $\{\boldsymbol{F}\}_{6(n+1) \times 1}^{e}$ in equation (3) is considered as the external force required for each elastic thin rod. The total finite element analysis is carried out by combining the infinitesimal rigid segment nodes and the elastic thin rod elements together. The external force required for each node is as follows:

$$
\{\boldsymbol{Q}\}=\sum_{i=1}^{n+1}\left\{\boldsymbol{F}^{b}\right\}^{i}+\sum_{e=1}^{n}\{\boldsymbol{F}\}^{e}
$$

Where, $\{\boldsymbol{Q}\}$ is the external force on the node, which has $6(n+1)$ components.

\section{Numerical simulation}

\subsection{Solving parameter setting}

The airflow velocity distribution in the twisting airflow chamber of jet vortex spinning is shown in Figure 3. We treat the hollow spindle as a relatively fixed wall. We consider the free-end fiber as an elastic thin rod, which is wrapped around the core fibers under the rotating airflow force $F$ in the twisting airflow chamber of jet vortex spinning. The head constraint of the free-end fiber is regarded as the fixed constraint. The rotating airflow force is regarded as the load on the elastic thin rod. Then, the static balance form of the fiber is calculated and analyzed under the given load.

The whole elastic thin rod is divided into $n$ units by the finite element method. The force of the airflow field, which is continuously distributed on the fiber surface, is simplified to their nodes. The contact constraint between the elastic thin rod and the wall is dealt by "the penalty function method"..$^{13}$ In the penalty function method, a repulsive force is exerted between the elastic thin rod and the wall. When the distance between the elastic thin rod and the wall is less, the repulsive force rapidly increases, and when the distance between the elastic thin rod and the wall is more, the repulsive force rapidly decreases to zero. The penalty function method can prevent the elastic thin rod from entering the wall during the step loading process. The fiber wrapped static equilibrium form is obtained by MATLAB programming and calculation, which is shown in Figure 4.

\subsection{Movement speed of free-end fiber}

The distribution of the free-end fiber velocity from the numerical simulation results is shown in Figure 5 . The fiber velocity increases gradually with $H$ and decreases at $H=5.25 \mathrm{~mm}$. Due to the special structure of the cone-shaped curved surface

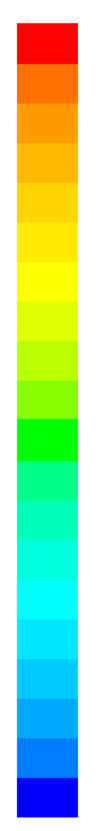

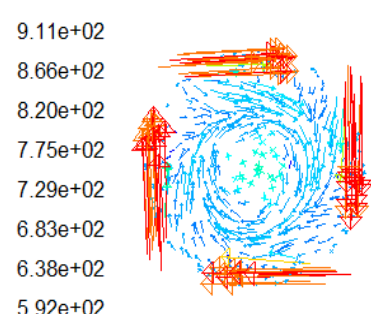

$5.47 \mathrm{e}+02$

$5.01 \mathrm{e}+02$

$4.56 \mathrm{e}+02$

$4.10 \mathrm{e}+02$

$3.65 \mathrm{e}+02$

$3.19 \mathrm{e}+02$

$2.73 \mathrm{e}+02$

$2.28 \mathrm{e}+02$

$1.82 \mathrm{e}+02$

$1.37 \mathrm{e}+02$

$9.12 \mathrm{e}+01$

4. $56 \mathrm{e}+01$

$6.16 \mathrm{e}-02$

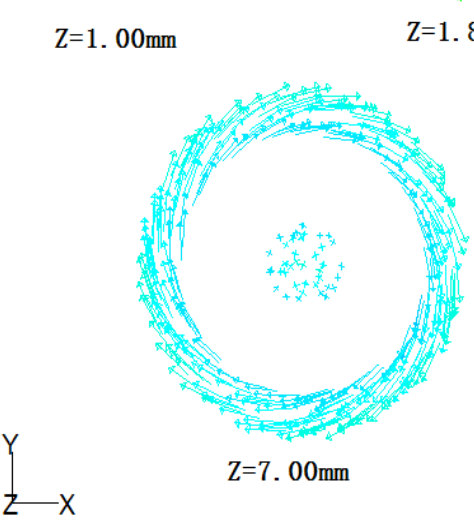

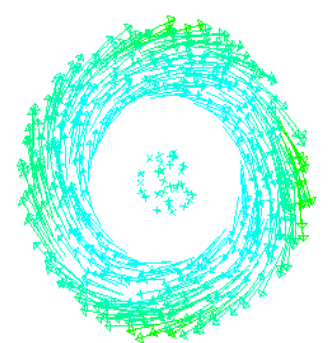

$Z=3.00 \mathrm{~mm}$

Figure 3. The airflow velocity on the planes of different Z-coordinates in the air-jet twisting zone. 


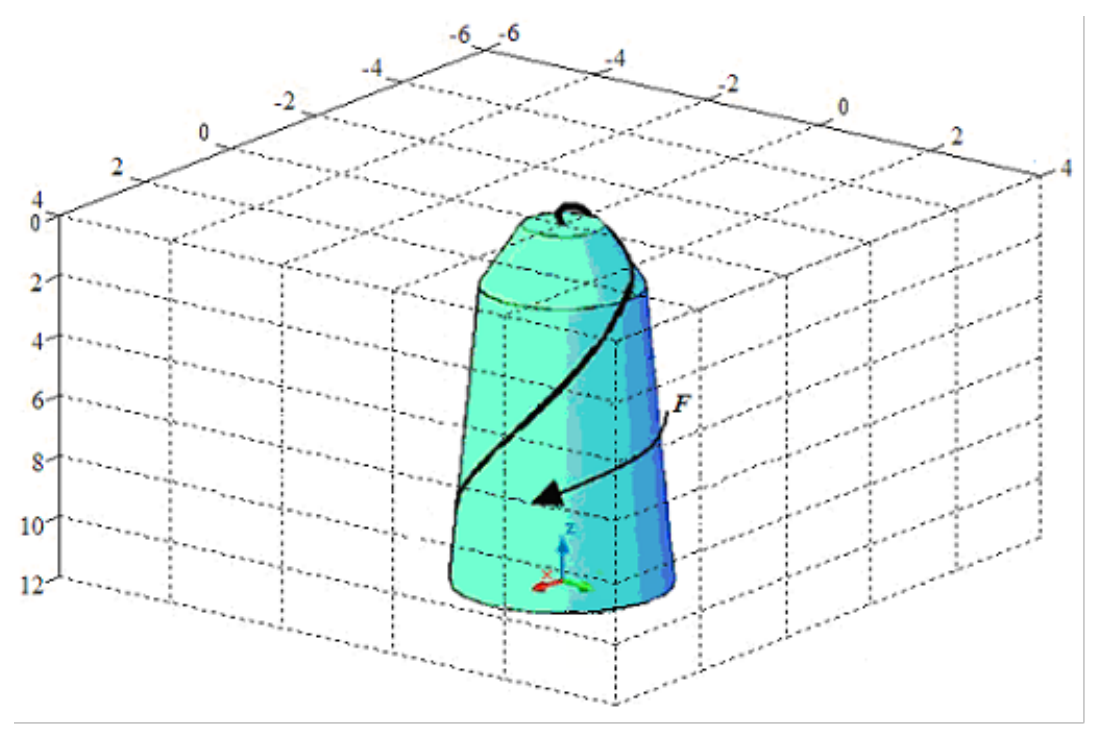

Figure 4. The trajectory of the free-end fiber around the top of the hollow spindle.

of the hollow spindle, the inclination angle of the cone-shaped curved surface of the hollow spindle is significantly changed at the plane position of $H=5.25 \mathrm{~mm}$. The position of the lodging fiber axis is determined by the inclination angle $d$ of the coneshaped curved surface of the hollow spindle. In this paper, the speed of the point on the fiber axis was fitted by Origin 8.5 software, and the expression formulae of the fiber velocities in different positions and different directions were obtained.

Based on the average motion model of particles, ${ }^{[13,14]}$ the velocity $v T a$ in the tangential direction of the free-end fiber flexible body at different positions is calculated as follows:

$$
v_{\text {Та }}= \begin{cases}223.93-\frac{180.24}{1+\exp (2.18 \cdot H-2.97)} & H<5.25 \mathrm{~mm} \\ -1.65+1023.89 \cdot \exp \left(-\frac{H}{3.34}\right) & H \geq 5.25 \mathrm{~mm}\end{cases}
$$

In the range of $H \leq 5.25 \mathrm{~mm}$, the velocity $v R a$ in the radial direction of the free-end fiber flexible body at different positions is not negligible. In the range of $H^{3} 5.25 \mathrm{~mm}$, the velocity $v R a$ in the radial direction of the free-end fiber flexible body at different positions is calculated as follows:

$$
v_{\mathrm{Ra}}=\left\{\begin{array}{cc}
0 & H<5.25 \mathrm{~mm} \\
3.44+\frac{45.56}{1+\exp (1.70 \cdot H-12.17)} & H \geq 5.25 \mathrm{~mm}
\end{array}\right.
$$

The velocity $v A a$ in the axial direction of the free-end fiber flexible body at different positions is calculated as follows:

$$
v_{\text {Aa }}= \begin{cases}257.32-\frac{6121.98}{1.81 \cdot \pi(H-1.81)^{2}+7.25 \pi} & H<5.25 \mathrm{~mm} \\ -12.41+1071.19 \cdot \exp \left(-\frac{H}{2.51}\right) & H \geq 5.25 \mathrm{~mm}\end{cases}
$$

\subsection{Trajectory of free-end fiber}

It is assumed that the trajectory of the free-end fiber is an isvel angle conical helix. Based on the isvel angle conical helix

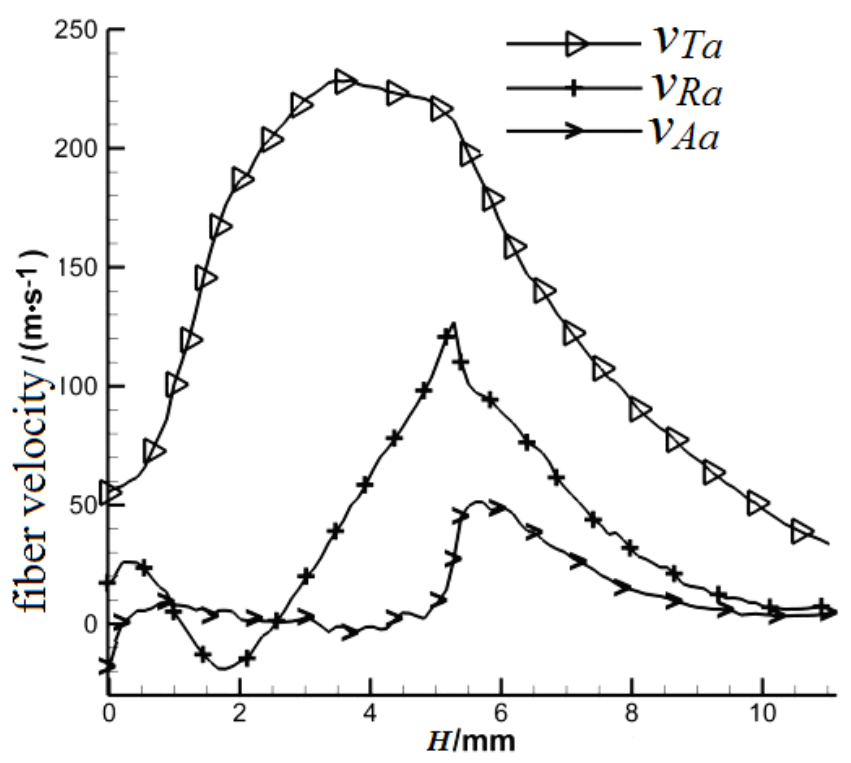

Figure 5. The distribution of the free-end fiber velocity.

theory, $[13,14]$ in the range of $0 \mathrm{~mm}<H \leq 5.25 \mathrm{~mm}$, the space position equation of infinitesimal rigid segment is as follows:

$$
\left\{\begin{array}{l}
x(\varphi)=\left(H \cdot \sin \delta+r_{0}\right) \cos \varphi \\
y(\varphi)=\left(H \cdot \sin \delta+r_{0}\right) \sin \varphi \\
z(\varphi)=H
\end{array}\right.
$$

In the range of $5.25 \mathrm{~mm} \leq H<11.15 \mathrm{~mm}$, the space position equation of infinitesimal rigid segment is as follows:

$$
\left\{\begin{array}{l}
x(\varphi)=\left[(H-5.25) \cdot \sin \delta+r_{1}\right] \cos \varphi \\
y(\varphi)=\left[(H-5.25) \cdot \sin \delta+r_{1}\right] \sin \varphi \\
z(\varphi)=H
\end{array}\right.
$$

Where, $\varphi$ is the isvel factor of the conical helix; $r_{1}$ is the outer contour radius of the hollow spindle in the plane at $H=5.25 \mathrm{~mm}$; 
$d$ is the inclination angle of the cone-shaped curved surface of the hollow spindle, in the range of $0 \mathrm{~mm}<H \leq 5.25 \mathrm{~mm}, \mathrm{~d}=20^{\circ}$; in the range of $5.25 \mathrm{~mm} \leq H<11.15 \mathrm{~mm}, \mathrm{~d}=60^{\circ}$.

The trajectory function of the free-end fiber in the high-speed rotary motion on the cone-shaped curved surface of the hollow spindle is deduced as follows:

$$
d l=\sqrt{x(\varphi)^{2}+y(\varphi)^{2}+z(\varphi)^{2}}
$$

According to the trajectory of the free-end fibers in the twisting airflow chamber of jet vortex spinning, in the range of 0 $\mathrm{mm}<H \leq 5.25 \mathrm{~mm}$, the angle between the fiber axis and the baseline on the hollow spindle cone-shaped curved surface $a_{1} » 53.5^{\circ}$; in the range of $0 \mathrm{~mm}<H \leq 5.25 \mathrm{~mm}$, the angle between the fiber axis and the baseline on the hollow spindle coneshaped curved surface $a_{1} » 59.3^{\circ}$.

\section{Experiments}

The jet vortex spinning speed is $300-500 \mathrm{~m} / \mathrm{min}$. The twisting airflow chamber of jet vortex spinning is non-visible, and so it cannot observe the actual movement of the free-end fiber through the imaging device. It can be seen from Figure 6 that the motion of the free-end fiber flexible body under the high-speed rotating airflow in the twisting airflow chamber is consistent with the space morphological conformation of the fiber flexible body in the yarn. Therefore, the motion characteristics of the free-end fiber flexible body can be transformed into the space morphological conformation of the fiber flexible body in the yarn structure.

\subsection{Experiments design}

The spinning process parameters are listed in Table 1. The production equipment is MVS860. The raw material is viscose fiber, which is $38 \mathrm{~mm}$ '1.33 dtex and produced by Leading Fiber Innovation. The quantity of the sliver is $21 \mathrm{~g} / 5 \mathrm{~m}$; the unevenness of the rough brands is $\leq 2 \%$; and the moisture regain of the rough brands is $11.0 \%$. The spinning speed is $360 \mathrm{~m} / \mathrm{min}$. The environment of the workshop is under standard atmospheric; the relative humidity is $(65 \pm 3) \%$; the temperature is $(20 \pm 2)^{\circ} \mathrm{C}$, according to $\mathrm{FZ} / \mathrm{T} 1086-2000, G B / T 398-2008$, and GB/T5324-2009.

The specimen was tested using a DIGITAL MICROSCOPE $\mathrm{KH}-7700$, according to $\mathrm{GB} / \mathrm{T} 398-2008$. Before testing, the specimens should be put into $125-135^{\circ} \mathrm{C}$ saturated steam for 20-30 min, to get the heat treatment. The heat treatment can help the yarn keep the original shape, without being changed Table 1. Spinning process parameters

\begin{tabular}{|c|c|c|c|c|c|}
\hline $\begin{array}{c}\text { Process } \\
\text { parameters } \\
\text { (dtex) }\end{array}$ & $\begin{array}{c}\text { Nozzle pressure } \\
\text { (MPa) }\end{array}$ & $\begin{array}{c}\text { Main draft } \\
\text { multiple }\end{array}$ & $\begin{array}{c}\text { Distance } \\
\text { between front } \\
\text { roller jaw and } \\
\text { hollow spindle } \\
\text { entrance (mm) }\end{array}$ & $\begin{array}{c}\text { Roller center } \\
\text { distance } \\
\text { (mm'mm) }\end{array}$ & $\begin{array}{c}\text { Hollow spindle } \\
\text { inlet diameter } \\
\text { (mm) }\end{array}$ \\
\hline 19.4 & 0.55 & 35 & 20 & $43^{\prime} 45$ & 1.1 \\
\hline 14.5 & 0.55 & 44 & 20 & $43^{\prime} 45$ & 1.1 \\
\hline
\end{tabular}

by the external forces. The tested specimens are six bobbins when tested. The magnification of the yarns is 160 times. The main morphological characteristics of the yarns observed are shown in Figures 7 and 8.

\subsection{Experiment analysis}

As we can see, the main morphological characteristics of the yarns observed are shown in Figures 7 and 8 . The jet vortex spun yarn has a similar appearance of the ring spun yarn. The figures show that the wrapping angle $q$ of the regular wrapping fiber on the surface of the jet vortex spun yarn is consistent with the results of the numerical simulation based on the elastic thin-rod finite element model. So, in this paper, it is feasible for the elastic thin rod element model to be used to simulate the trajectory of free-end fiber under the high-speed rotating airflow in the twisting airflow chamber of jet vortex spinning

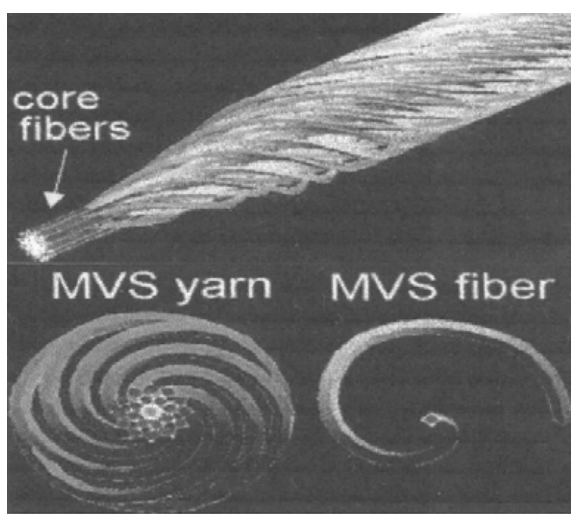

Figure 6. The ideal structure of jet vortex spun yarn.

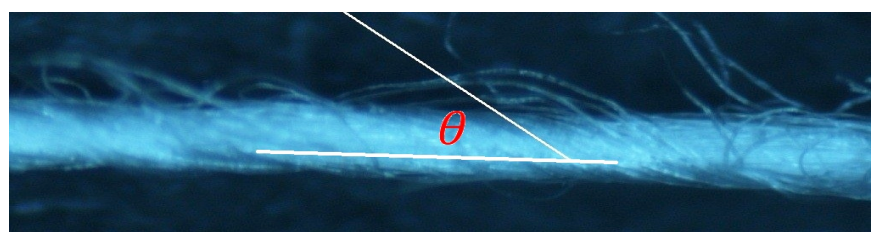

Figure 7. The surface structure of 19.4 dtex jet vortex spun yarn.

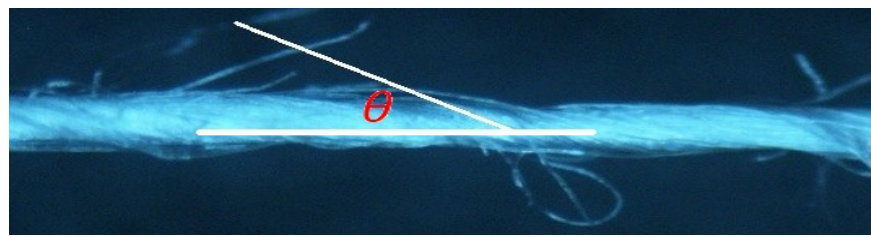

Figure 8. The surface structure of $14.5 \mathrm{dtex}$ jet vortex spun yarn. from different spindles that have 10 replications per bobbin 


\section{Conclusion}

In this paper, we analyzed the static equilibrium state of the free-end fiber during the jet vortex spinning airflow twisting process and established the elastic thin rod element model of flexible fiber. We numerically simulated the geometric large deformation process of the elastic thin rod element free-end fiber model and obtained the movement law of the free-end fiber under the high-speed rotating airflow. Finally, the experiments were done to test the theoretical results of the finite element simulated fiber trajectory. In this paper, the elastic thin rod element model of flexible fiber is feasible and solves the problem that the finite element analysis method of rod element is difficult to be used in the actual calculation of three-dimensional large space deformation. By changing the initial conditions of the numerical simulation, the position of the free-end fiber can be determined, which provides a theoretical basis and a new research idea for the study of the process and principle of jet vortex spinning.

\section{Acknowledgments}

This work was supported by National Key R\&D Program of China (2017YFB0309200), Natural Science Foundation of Jiangsu Province (BK20180582), Jiangsu Postdoctoral Research Funding Scheme (2018K019A) and Fundamental Research Funds for the Central Universities (JUSRP11801).

\section{References}

[1] Beceren, Y., Nergis, B. U. (2008). Comparison of the effects of cotton yarns produced by new, modified and conventional spinning systems on yarn and knitted fabric performance. Textile Research Journal, 78(4), 297-303.

[2] Basal, G., Oxenham, W. (2006). Effects of some process parameters on the structure and properties of vortex spun yarn. Textile Research Journal, 76(6), 492-499.

[3] Tyagi, G. K., Sharma, D., Salhotra, K. R. (2004). Processstructure-property relationship of polyester-cotton MVS yarns: part $i$ - influence of processing variables on yarn structural parameters. Indian Journal of Fibre \& Textile Research, 29(4), 419-428.
[4] Li, Y. X., (2005). Jet vortex spinning exploration. China Textile Leader, (8), 69-67.

[5] Yuan, L. C., Li, X. R., Guo, Z., Jiang, X. M. (2018). Research progress in influence of vortex spinning nozzle on flow field. Journal of Textile Research, 39(1), 169-178.

[6] Ortlek, H. G., Nair, F., Kilik, R., Guven, K. (2008). Effect of spindle diameter and spindle working period on the properties of $100 \%$ viscose MVS yarns. Fibres \& Textiles in Eastern Europe, 16(3), 17-20.

[7] Liu, Y., Xu, L., (2006). Controlling air vortex in air-vortex spinning by zeng-he mode. International Journal of Nonlinear Sciences and Numerical Simulation, 7(4), 389392.

[8] Guo, H. F., An, X. L., Yu, Z. S., Yu, C. W. (2008). A numerical and experimental study on the effect of the cone angle of the spindle in murata vortex spinning macgine. ASME Journal of Fluids Engineering, 130(3), 1-5.

[9] Zou, Z. Y., Liu, S. R., Zheng, S. M., Cheng, L. D. (2010). Numerical computation of a flow field affected by the process parameters of murata vortex spinning. Fibres \& Textile in Eastern Europe, 18(2), 35-39.

[10] Liu, Y. Z. (2006). Nonlinear mechanics of elastic thin rods. (1ed). Tsinghua University Press (Beijing).

[11] Hong, J. Z., (1999). Calculation of multi-system dynamics. (1ed). Higher Education Press (Beijing).

[12] Xia, D. L. (1990). The forming principle of isometric conical helix and the drawing method of projection. Journal of Hefei University of Technology (Natural Science), 13(3), 99-106.

[13] Xia, D. L. (1992). Geometric Characteristics and mapping method of conical spiral stepped helix. Journal of Hefei University of Technology (Natural Science), 15(4), 138145.

[14] Xu, S. D., Wang, X. (2012). Discussion on two methods evaluation yarn evenness. Cotton Textile Technology, (12), 29-31. 